\title{
Nuclear energy generation, numerical simulation accuracy and nuclear data
}

\author{
D. Lecarpentier ${ }^{\mathrm{a}}$ \\ EDF R\&D, 1 av. du Général de Gaulle, 92141 Clamart, France
}

\begin{abstract}
In the French context, it appears that the accuracy of the nuclear data comply with the utility need. However, a constant reduction of the calculation uncertainties is achieved with more precise physics and numerical methods and improved nuclear data. The aims for this uncertainty reduction are: to increase the competitiveness, by reduced operating conservatisms in order to extend the reactor lifespan and by optimising the existing series of nuclear plants and associated fuel cycle: extended burn-up, MOX fuel, reduced damage to the reactor vessel... and to evaluate and to develop the future nuclear energy systems (Generations III and IV) with a sufficient accuracy.

The nuclear reactor operation is currently supported by numerical simulation: if the discrepancies between precalculated values and measures in operation are sufficiently low, the required authorisations are given by the Safety Authority. EDF core calculations have been relying on JEF2.2 for more than fifteen years. This association of code and database gives a satisfying uncertainty level for the current reactors and associated fuel management, but the development of a new calculation scheme named N3C-V2 has been decided at EDF, relying simultaneously on: improved numerical scheme allowed by today's powerful computers and recent physics improvements; - a new set of nuclear data: JEFF3.1. This work is considerable, and the expected results are the reduction of uncertainty on the main parameters of future optimised fuel management in existing reactors and in EPR.

Concerning Gen IV reactors and actinide transmutation, progresses are still needed (even for Sodium cooled FBR which are the reference Gen IV solution of the industrial EDF), mainly in the fast spectrum. The aim is to evaluate with a better accuracy: Fuel evolution, in particular, the formation of Minor Actinides (Am, Cm, Np) or important isotopes, like ${ }^{232} \mathrm{U}$ in the thorium cycle; Breeding gain ${ }^{238} \mathrm{U}$ and ${ }^{232} \mathrm{Th}$ captures, ${ }^{239} \mathrm{Pu}$ and ${ }^{233} \mathrm{U}$ fissions, in the fast range for ${ }^{238} \mathrm{U}$ and ${ }^{239} \mathrm{Pu}$, in all ranges for ${ }^{232} \mathrm{Th}$ and ${ }^{233} \mathrm{U}$ ); Doppler effect; Void effect in fast spectrum reactors is a major issue and can be influenced by the heavy nuclei cross sections, but also by the elastic and inelastic scattering cross sections of the coolant (scattering of $\mathrm{Na}$ or $\mathrm{Pb}$ for ex.). Finally, uncertainty on cross-sections must become available in the evaluation files, for they will help to differentiate the part of uncertainty coming from the data and from the calculation methods.
\end{abstract}

\section{Introduction}

The definition of nuclear data need by utilities using neutronic codes is a regularly repeated exercise. It is reassessed when new data evaluations are made available and when the industrial practices in the nuclear fleet management change (fuel burn-up extension, new fuel management...). The time scale of this process is large because:

- The development time of a new nuclear data evaluation is very long (15 years between JEF2 and JEFF3);

- The industrial calculation scheme is significantly impacted so it has to be modified and be completely re-qualified;

- The industrial fleet management evolves slowly (and so do the new data needs).

That's why the needs are now approximately the same as in year 2001, when such an exercise had been realised by the CFDN, refs. [1,2] (Comité Français des Données Nucléaires). The reason for that is that the new library JEFF3.1 has been released in 2005, so that EDF's industrial

\footnotetext{
${ }^{a}$ e-mail: david-n.lecarpentier@edf.fr
}

calculation scheme is still the one developed in the 90's, relying on JEF2 evaluation.

A new neutronic calculation scheme called N3C-V2 is now developed by EDF, with a large contribution of the CEA, relying on JEFF3.1 evaluation, which has taken into account most of the analysis of JEF2.2, for example in [2] and [3]. This scheme aims to reduce the calculation uncertainties, in order to reduce the unnecessary conservatisms especially in the preparation of the anticipated evolutions/optimisations of the French PWR fleet: burn-up and uranium enrichment increase, MOX fractions increase in core, higher Plutonium enrichment in the MOX fuel, use of uranium reprocessed from spent fuel (with limits in ${ }^{232} U$ et ${ }^{236} U$ to consider), burnable poisons $(\mathrm{Gd}, \mathrm{Er}) .$. , new absorbers (Hf, $\mathrm{HfB}_{2}$, $\mathrm{Eu} . .$.$) , clad with new doping isotopes (Nb, Va, Si...), new$ fuel management allowed by EPR... All these evolutions intend to increase the competitiveness, the availability and flexibility of the nuclear generation fleet.

Finally, the utility EDF has to prepare the long term evolution of the fleet, with the possible apparition of Gen IV reactors in the next decades. Feasibility studies of Gen IV reactors, mostly Sodium cooled Fast Breeder Reactors, needs also a sufficient knowledge of nuclear data for specific isotopes and neutron spectra. 


\section{Industrial needs for PWR operation}

\subsection{Nuclear data used in support to PWR operation}

In this section, we give qualitatively the main physical parameters involved in the nuclear production and impacted by the nuclear data.

\subsubsection{Safety and operation}

- Safety coefficients (Doppler, water temperature, delayed neutron fractions...);

- Residual power;

- Protection.

To be correctly assessed, all these parameters imply a good knowledge of all isotopes the cross-sections values, but also the decay constants, branching ratios and fission yields.

\subsubsection{Reactor life time/damages to the materials (Vessel/internals)}

DPA, Helium production, n,p/alpha sections, inelastic diffusion.

\subsubsection{Nuclear material management (transport, reprocessing, waste disposal)}

The cross-sections, fission yields, decay constants, branching ratios are also necessary to compute as exactly as possible the fuel composition at each step of its life cycle.

The radioactive emissions have also to be computed at different time scales for the transport, the reprocessing and the final disposal of wastes.

\subsection{Challenges associated to the uncertainties}

\subsubsection{How uncertainties are taken into account by the industrials}

The role of industrials is to develop, use and dismantle their installations in the most efficient way, while meeting the safety requirements.

For the core conception, a list of criteria has to be satisfied, especially concerning the good behaviour of the first barrier (fuel clad). The three safety functions have to be met:

1. reactivity control,

2. fission Products confinement,

3. residual power evacuation.

Among the different safety design constraints, the following can be cited:

- No fuel inner melting;

- Surface heat flux through the clad < critical flux;

- Sub-criticality of the installations when they are stopped, with vessel closed $\left(\mathrm{k}_{\mathrm{eff}}<0.95\right)$;

- Limit reject rates.
These safety constraints are verified if:

Computed value + uncertainties + margins < criteria .

For the reactor exploitation, the situation is slightly different: the Safety Authority allows the exploitation if the difference between pre-computed values and measured values stays sufficiently low. Some examples:

- the critical boron concentration $(<50 \mathrm{ppm})$;

- the rods reactivity weight $(<10 \%)$;

- the temperature coefficient $(<0)$ (conservative).

This is resumed by the relationship:

Computed value - measured value < criteria.

As a consequence, an imperfect knowledge of the uncertainties will cause:

For the conception, unnecessary over-sizing, which is economically penalizing,

For the exploitation, a higher probability of criteria overshoot, causing a reduction of the reactor availability. In fact, it can then be necessary to perform justification studies with more sophisticated tools and the utility can not run the reactor in the initially planed configuration.

\subsubsection{Origin of the uncertainties}

The uncertainties come from:

- Computation methods;

- Nuclear data;

- System modelisation, non neutronic parameters (densities, sizes, initial fuel composition...).

The uncertainty related to the methods can be understood by comparison with more precise and sophisticated models, with the same nuclear data: fine deterministic transport calculation or Monte Carlo calculation. This type of uncertainty can be computed and taken into account with specific uncertainties and/or adjustment coefficients in case of systematic bias.

The uncertainty due to the nuclear data can be reduced only by improving the knowledge of the nuclear (crosssections, fission yields, decay constants, emission spectra, delayed neutron fractions, etc).

Different means of improving the data can be used:

- Reducing the errors due to the experimental methods;

- Measurement of more precise differential data, for the most important isotopes and the adequate neutron velocities;

- Better evaluation of the available set of data;

- A qualification on a large set of integral experiments, with a research of needed improvements, based on a coherent statistical adjustment, leading to correcting factors, or to new evaluations.

\subsubsection{Potential gains linked to the uncertainty reduction}

We give in this paragraph some orders of magnitude of potential gains affecting a nuclear fleet thanks to a better uncertainty's knowledge (we consider an hypothetic reactor fleet of 50 units of $1000 \mathrm{MWe}$ ): 
A $1 \%$ underestimated cycle length, due to an underestimation of approximately $0.1 \%$ of the production cross-section $\boldsymbol{v} \boldsymbol{\sigma}_{f}$ would lead to an annual loss of energy equivalent to $1 / 2$ fuel core (without taking into account the price of the substitution energy).

The increase of $1 \%$ of the linear power leads also to a $1 \%$ gain of the fleet power, that means $1 / 2$ a reactor's power on the whole.

A gain on the residual power on the short time scale will cause a faster opening of the reactor vessel and improve the availability of all the reactors. The order of magnitude of the non production of the whole fleet during one day is equivalent to the cost of $1 / 2$ a reactor fuel.

So in conclusion, utilities have a significant interest in improving the uncertainty's knowledge, and reducing it. This reduction could lead to reduced unnecessary conservatisms, to put the safety where it is really necessary, and then result to economic gains.

\subsubsection{Target precisions for the long term}

The target uncertainties are the same as in 2001, because they are ambitious long term objectives. It is not guarantied that the scheme in development will reach those performances.

The target uncertainties are (with $2 \sigma$ precision):

Nuclides density evolution (end of irradiation concentration):

Isotopes with important reactivity weight $+/-2 \%$

Other isotopes $+/-10 \%$

Cycle length $+1-3 \%$

Boron concentration $\quad+/-10 \mathrm{ppm}$

Rod's weight $+1-5 \%$

Local power $\quad+/-5 \%$

Residual power $\quad+/-5 \%$

Water temperature coefficient $\quad+/-1 \mathrm{pcm} /{ }^{\circ} \mathrm{C}$

Doppler coefficient $\quad+/-10 \%$.

\section{Ongoing efforts for PWR calculation improvement}

To choose the data that have to be improved, one has to know the basic nuclear data sensitivity of the parameters used for the conception and the exploitation.

\subsection{New evaluations}

The qualification of JEF2.2 data set has lead to recommendations on the main nuclear data to be improved (see ref. [3]). Those recommendations were followed when establishing JEFF3.1.

The qualification of JEFF3.1 has begun, particularly at the CEA (see section 3.3 and refs. [5-7]). The integral data of the main actinides show calculation experiment errors less than $2 \%$, except for ${ }^{234} \mathrm{U}$ and ${ }^{237} \mathrm{~Np}$ the evaluation of which has to be reassessed because of an error order of magnitude of $10 \%$, ref. [5].

\subsection{New calculation schemes}

An industrial reactor calculation scheme is a rather complex object, with errors compensations at its different levels. The principle of such a calculation scheme is the following:

1. generation of multi-group cross sections based on the original point wise evaluation (99 groups and JEF2 today, 281 groups and JEFF3.1 in the near future);

2. APOLLO2 multi-group transport assembly calculation (99 groups $\mathrm{CP}^{1}$ multicell methods now, 281 groups $\mathrm{CP}$ chained with 26 groups MOC $^{2}$ in the future). Selfshielding methods will also be improved;

3. two groups condensation, homogenisation and transportdiffusion equivalence;

4. two groups core calculation (homogeneous diffusion today and pin by pin tomorrow).

Now, the JEFF-3.1 data are being qualified by the CEA on the two first stage, while EdF is developing the two last stages. The whole scheme, called N3C-V2 has to be qualified at the 2013 horizon, so that it is now too early to evaluate the precision gains due to JEFF-3.1 and to give new recommendations for further cross sections improvements.

\subsection{Integral experiments in support to the qualification}

Four types of experiments stand in support to the reactor calculation scheme qualification:

- Fundamental experiments (like oscillations of capturing isotopes OCEAN and Minor Actinides OSMOSE in one of the CEA experimental reactor MINERVE, refs. [6,7];

- Mock-ups experiments (at the CEA: EOLE, MASURCA [7]);

- Analysis of irradiated PWR pins (dissolutions). The discrepancies between calculation with JEF2.2 and measure has lead to numerous recommendations for JEFF3.1 [3];

- Reactivity evolution with the burn-up (PWR irradiated fuel oscillations in Minerve, and in line reactivity control of PWRs).

Progressively, the qualification area of neutronic codes (APOLLO2) is beeing extended (for MOX parity, High BurnUps MOX, Absorbing isotopes Er, Hf, Gd, "grey" control rods...).

\section{Nuclear data needs for future reactors}

The study of Gen IV concerns mostly the R\&D today, except for sodium cooled fast reactors which exist at the industrial scale in some countries (BN-600 in Russia, Phénix and SuperPhénix in France, Monju in Japan, PFBR 500 MWe in construction in India).

Two phases in the conception of Gen IV reactors are being considered by most of the publications: "viability" and

\footnotetext{
${ }^{1}$ Collision Probability method.

2 Method Of Characteristics.
} 
Table 1. Uncertainty target for "viability" and "performance" [10].

\begin{tabular}{ccc}
\hline Parameter & viability & performance \\
\hline $\mathrm{k}_{\text {eff }}(\mathrm{BOL})$ & $0.7 \%$ & $0.3 \%$ \\
Local power density & $5 \%$ & $3 \%$ \\
Reactivity swing & $1 \%$ & $0.5 \%$ \\
Structure damage & $15 \%$ & $9 \%$ \\
Void effect & $16 \%$ & $10 \%$ \\
Doppler effect & $16 \%$ & $10 \%$ \\
Control rod worth & $16 \%$ & $10 \%$ \\
\hline
\end{tabular}

"performance" phases. The uncertainties (one sigma) level requested for the two phases are (ref. [10]):

\subsection{Data needs for the different Gen IV reactors}

\subsubsection{Fast Breeder Reactors (FBR)}

In comparison with the other Gen IV reactors, there is a large experimental feedback from the FBR operation. That is particularly true for the sodium cooled FBR with oxide fuel, which represents for EDF the reference Gen IV industrial choice.

In fast neutron spectrum, the multi-group cross-sections are adjusted by a least-square like process to a great number of integral experiments (approximately 400 for the CEA code ERANOS $[17,13]$ whose adjusted library ERALIB1 allows a substantial uncertainty diminution compared to the unadjusted JEF-2.2 library).

Efforts are still needed in order to diminish the uncertainties on the principal parameters of these reactors (even if the "performance" phase uncertainties were fulfilled for the Super-Phénix plant). In fact, new configurations are now studied for the Gen IV SFR, which certainly need more integral qualification:

FBR Na with dense fuels: UPuN, UpuC, UpuZr;

FBR Na with a sodium upper plenum which aims at reducing the void effect;

Gaz cooled FBR (Helium) with UPuC fuel and SiC cladding;

$\mathrm{Pb}$ and $\mathrm{Pb}-\mathrm{Bi}$ cooled FBR: for these reactors, there is a first Russian qualification basis (integral experiments in the BFS zero-power reactor ref. [12]). Adjustment of the multigroup cross-sections to those integral measurements seems to be necessary because large discrepancies have been shown in ref. [15] on important parameters like the void effect between ERANOS, which did not use a $\mathrm{Pb}$ adjusted library and TRIGEX (Russian FBR code) which used a Pb adjusted library.

So, in the near future, the question will be raised of generating JEFF3.1 based multi-group libraries adjusted on the same integral experiments ref. [17].

Finally, in all the FBR, a Minor Actinide multi-recycling either in homogenous mode or in blankets is envisaged by some R\&D organisations. So it is necessary to know well the MA cross-sections in fast spectrum in order to compare precisely those options of MA management to their direct disposal option.

\subsubsection{High conversion ratio water cooled reactors}

Those reactors generally have a harder spectrum than today's water cooled reactors because they are under-moderated in order to favour the regeneration of ${ }^{238} \mathrm{U}$ into ${ }^{239} \mathrm{Pu}$.

Integral experiments have already been conducted in the past in this kind of epithermal spectrum. Minor actinides and capturing isotopes oscillations are foreseen by the CEA in the MINERVE reactor in the next years (MORGANE-R ref. [7]).

Basic data linked to the Thorium cycle $\left({ }^{232} \mathrm{Th},{ }^{233} \mathrm{U},{ }^{233} \mathrm{~Pa}\right)$ are also important for all kinds of spectra because there is a number of high conversion ratio reactors designs $\left({ }^{232} \mathrm{Th}\right.$ to $\left.{ }^{233} \mathrm{U}\right)$ : Shippingport under-moderated reactor in the US ref. [18], heavy water cooled Thorium reactor foreseen in India, ref. [19]).

In these reactors like in others, ${ }^{232} \mathrm{U}$ ways of formation have to be particularly mastered because highly penalising gamma emissions (fabrication, reprocessing) are associated with ${ }^{232} \mathrm{U}$ and its decay family.

\subsubsection{High temperature reactors (HTR)}

(V)HTR are one of the six Gen IV reactors. There is a large variety of HTR, depending on the graphite moderation (moderation ratio) and the fuel management (enriched Uranium, Plutonium burner with very high burn-up or even Thorium fuelled HTR with high conversion ratio). For each of these types of HTR, cross-sections in different epithermal spectra are needed, see ref. [2]. One element specifically used in the HTR is the Erbium, used as burnable poison and to improve the graphite temperature coefficient. There is also an experimental feedback from HTR's developed in the 70's, so that the uncertainties are not too high on this type of reactor.

\subsubsection{Molten salt reactors (MSR)}

Different types of breeder MSR concepts are available today:

- ${ }^{233} \mathrm{U}-{ }^{232} \mathrm{Th}$ fuelled MSR with fluoride salt a thermal neutron spectrum, moderated by graphite, refs. [22,23];

- ${ }^{233} \mathrm{U}_{-}{ }^{232} \mathrm{Th}$ fuelled MSR with fluoride salt an fast neutron spectrum, promoted by the CNRS, ref. [21];

- ${ }^{238} \mathrm{U}-\mathrm{Pu}$ fuelled MSR with chloride salt a fast neutron spectrum, ref. [20].

For these reactors to, the improvement of Thorium chain isotopes at all energies from $0.1 \mathrm{eV}$ to $10 \mathrm{MeV}$ will reduce the design uncertainties [14].

The knowledge of diffusion and capture cross-section of graphite and potential constituents of the salt is also needed: $\mathrm{Li}, \mathrm{F},{ }^{35} \mathrm{Cl}$ et ${ }^{37} \mathrm{Cl}, \mathrm{Na}, \mathrm{Be}, \mathrm{K}$ either in fast or in thermal spectrum. Today precision is however certainly sufficient for the viability phase.

\section{Conclusions}

The nuclear data related efforts are very costly (man power, experimental means) and time consuming. The good scale for such efforts is the international scale. So a utility like EDF supports the efforts undertaken in Europe with the JEF 
evaluations. EDF takes also part to the CANDIDE European project (ref. [8]) for the definition of nuclear data R\&D effort related to future nuclear reactors.

The integral experiments and their interpretation have also to be shared as much as possible by nuclear stake holders, for the optimisation of Gen II and III, and for the conception of Gen IV reactors. From this point of view, ICSBEP and IRPhEP projects go in the right direction (ref. [16]).

The other effort to share at the international scale is the inclusion of variance-covariance data in the cross-section evaluations. This long term objective will certainly be reached in the next generation of evaluations.

\section{References}

1. F. Storrer et al., Priority Nuclear Data Needs for Industrial Applications, J. Sci. Technol., Suppl. 2, 1357 (2002).

2. D. Hittner, Industrial Perspective on Nuclear Data, J. Sci. Technol., Suppl. 2, 1344 (2002).

3. B. Roque et al., Experimental validation of the code system DARWIN for spent fuel isotopic predictions in fuel cycle applications, in Proceedings of the International Conference PHYSOR, 2002, Seoul, Korea.

4. JEFF Report 17, The JEF-2.2 Nuclear Data Library.

5. J.P. Hudelot et al., The OSMOSE Program for the qualification of Integral cross sections of Actinides: Preliminary Results in a PWR-Uox Spectrum, in Proceedings of the International Conference Physor 2006, Vancouver, Canada.

6. P. Fougeras et al., EOLE, MINERVE and MASURCA facilities and their associated experimental programs, in Proceedings of the International Conference ICONE13, 2005, Beijing, China.

7. M. Antony et al., The OSMOSE experimental program for the qualification of integral cross sections of actinides, in Proceedings of the International Conference ENC, 2005, Versailles, France.

8. J. Blomgren et al., CANDIDE - Coordination action on nuclear data for industrial development in Europe (these proceedings).

9. P. Fink, Future of Nuclear Energy and the role of nuclear data, in Proceedings of the International Workshop Nuclear Data Needs for Generation IV Nuclear energy Systems, 2005, Antwerpen, Belgium.

10. G. Rimpault, Nuclear Data Needs for the assessment of Gen IV Systems, in Proceedings of the International Workshop Nuclear
Data Needs for Generation IV Nuclear energy Systems, 2005, Antwerpen, Belgium.

11. G. Aliberti et al., Sensitivity of Advanced Reactor and fuel cycle performance parameters to nuclear data uncertainties, in Proceedings of the International Workshop Nuclear Data Needs for Generation IV Nuclear energy Systems, 2005, Antwerpen, Belgium.

12. V. Smirnov et al., The lead cooled fast reactor benchmark BREST-300: Analysis with sensitivity method, in Proceedings of the International Workshop Nuclear Data Needs for Generation IV Nuclear energy Systems, 2005, Antwerpen, Belgium.

13. A. Kochetkov et al, Benchmark-experiments for Pb and Bi neutron data testing, in Proceedings of the International Conference Physor 2006, Vancouver, Canada.

14. A. Bidaud et al., Sensitivity and uncertainty study for thermal molten salt reactors, in Proceedings of the International Workshop Nuclear Data Needs for Generation IV Nuclear energy Systems, 2005, Antwerpen, Belgium.

15. V. Smirnov et al., The lead cooled fast reactor benchmark BREST-300: Analysis with sensitivity method, in Proceedings of the International Workshop Nuclear Data Needs for Generation IV Nuclear energy Systems, 2005, Antwerpen, Belgium.

16. J.B. Briggs et al., The International Reactor Physics Experiment Evaluation Project, in Proceedings of the International Conference Physor 2006, Vancouver, Canada.

17. J. Tomasi, Present status of JEFF3.1 validation for fast reactors using the ERANOS2.1 code system, in Proceedings of the International Conference Physor 2006, Vancouver, Canada.

18. G.L Olson et al., Fuel Summary Report: Shippingport Light Water Breeder Reactor, Idaho National Engineering and Environmental Laboratory Report INEEL/EXT-98-00799.

19. R.B. Grover, Subhash Chandra, Scenario for growth of electricity in India, Energy Policy 34, 2834 (2006).

20. A. Mourogov, P. Bokov, Potentialities of the fast spectrum molten salt reactor concept REBUS-3700, in Proceedings of the International Conference ICENES 2005, Brussels, Belgium.

21. E. Merle-Lucotte et al., Fast Thorium Molten Salt Reactors started with Plutonium, in Proceedings of the International Conference ICAPP 2006, Reno, USA.

22. L. Mathieu, D. Heuer et al., The Thorium Molten Salt Reactor: Moving on from the MSBR (submitted to Prog. Nucl. Energy, 2006).

23. J. Vergnes, D. Lecarpentier, The AMSTER concept (Actinide Molten Salt TransmutER, Nucl. Eng. Design 216, 43 (2002).

24. Ph. Bioux, D. Biron (2001) (private communication). 